\title{
Comparación de métodos de exposición dermal a plaguicidas en una muestra de floricultores y productores de palmito y chayote en Costa Rica
}

\author{
Comparison of pesticides dermal exposure \\ methods in a sample of flowers, palm hearts \\ and chayote producers in Costa Rica
}

\author{
Ma. Lourdes Medina-Escobar' \\ Ma. Gabriela Rodríguez-Zamora² \\ Paula Zamora-Rodríguez ${ }^{3}$
}

2 Bachiller en Química, Escuela de Ingeniería en Seguridad Laboral e Higiene Ambiental, Instituto Tecnológico de Costa Rica, Cartago, Costa Rica.Tel. (506) 2550-2800. Correo electrónico: garodriguez@itcr.ac.cr

3 Ingeniera en Seguridad Laboral e Higiene Ambiental, Instituto Costarricense de Electricidad. Correo electrónico: pzamorar@ice.go.cr 


\section{María de Lourdes Medina escobar}

Originaria de la ciudad de David, Panamá, hizo sus estudios universitarios en la Universidad de Costa Rica, donde se graduó en Química y luego obtuvo un posgrado en Química Industrial.

Durante el curso de sus estudios superiores, trabajó como asistente en el Centro de Investigación en Contaminación Ambiental, donde dio sus primeros pasos hacia la investigación.

EITEC le abrió sus puertas en el 200 I, en la Escuela de Seguridad Laboral e Higiene Ambiental, con el fin de que se encargara del Laboratorio de Higiene Analítica que estaba siendo trasladado por medio de un convenio con el Instituto Nacional de Seguros (INS) para dar soporte a la investigación, docencia y extensión en temas de exposición laboral a agentes químicos.

Desde entonces, ha trabajado en el desarrollo y adaptación de metodologías de muestreo y análisis de diversos agentes químicos. Ha participado en diferentes proyectos de investigación tendientes a caracterizar la exposición de sustancias químicas en distintos tipos de actividades: polvo de madera, solventes orgánicos en pinturas e imprentas, isocianatos en pintura de vehículos, agroquímicos y humos metálicos en soldadura.
En el periodo 2005-2009, se desempeñó como Coordinadora de la Maestría en Salud Ocupacional, programa interuniversitario compartido con la Universidad Nacional.

Actualmente, además de los proyectos de investigación y el apoyo a la docencia, colabora con empresas nacionales tanto públicas como privadas en el asesoramiento de evaluación de exposición ocupacional a agentes ambientales químicos, biológicos y físicos, siendo responsable de establecer el diseño de los estudios de manera que respondan a las necesidades del cliente y cumplan con los requerimientos técnicos, elaboración de las propuestas metodológicas correspondientes, análisis de la información y los reportes que se entregan al cliente, todo ello cuidando que las diferentes etapas del estudio se realicen de acuerdo a los lineamientos establecidos, y que el producto final sea acorde con los más altos estándares de calidad.

Entre sus pasatiempos favoritos están la música, la lectura, actividades familiares y disfruta de la compañ́a de buenos amigos y del ambiente de montaña. Reside en Santo Domingo de Heredia, donde comparte con su esposo y su hijo. 


\section{Ma. Gabriela Rodríguez Zamora}

Nació en San José el 24 de setiembre de 1980. Cursó la primaria en la Escuela Central de Tres Ríos y la secundaria en el Liceo Mario Quirós Sasso, en San Diego de La Unión. En ambas instituciones se graduó con mención honorífica.

En 1998 ingresó a la Universidad de Costa Rica, a la carrera de Bachillerato y Licenciatura en Matemática y en 1999 se trasladó a la carrera de Bachillerato y Licenciatura en Química.

Siendo estudiante universitaria, trabajó como asistente becada en la Sección de Química Analítica Cuantitativa. En 1999 comenzó a impartir lecciones particulares de Química, Matemática y Física a estudiantes de colegio. Desde entonces y hasta el año 2009, estuvo preparando estudiantes para exámenes de convocatoria y para las pruebas de Bachillerato en Educación Media.

Obtuvo suTítulo de Bachiller en Química en agosto de 2005 y el 17 de julio de 2006 ingresó a trabajar en el TEC, particularmente en el Laboratorio de Higiene Analítica de la Escuela de Ingeniería en Seguridad Laboral e Higiene Ambiental, en donde se ha desempeñado como Oficial de Control de Calidad. En este laboratorio colabora en la implementación y control de métodos de análisis mediante las técnicas de cromatografía de gases, HPLC, gra- vimetría, espectrofotometría de absorción atómica y UV-Visible. También es responsable de la revisión de estrategias de muestreo e informes de resultados.

Con siete años y medio de ejercicio profesional como investigadora y docente universitaria, ha complementado su formación profesional inicial con cursos y seminarios en el área de Higiene Ocupacional. Además, ha participado en tres congresos internacionales y en seis proyectos de investigación orientados a la evaluación de riesgos higiénicos, ergonómicos y de seguridad en diversas actividades productivas a nivel nacional, tales como: el asfaltado de carreteras, la industria metalmecánica, labores agrícolas (en cultivos de palmito, chayote, helechos y flores), en el sector de la construcción, así como en la aplicación de lacas y pintura en mueblerías y talleres de enderezado y pintura de vehículos. También ha tenido la oportunidad de impartir los cursos de Teoría y Taller de Agentes Ambientales Químicos y Biológicos, pertenecientes a la carrera de Ingeniería en Seguridad Laboral e Higiene Ambiental del TEC.

Actualmente se encuentra cursando la Maestría en Salud Ocupacional con énfasis en Higiene Ambiental, ofrecida en conjunto por el TEC y la UNA, con el fin de reforzar su perfil académico y profesional. 


\section{Paula Zamora Rodríguez}

Buscando una posibilidad para conocer diferentes disciplinas, se abrió la puerta de la Salud y Seguridad Ocupacional, en definitiva, la mejor decisión fue entrar en ella.

En el año 1986 en el cantón de Pococí, específicamente en el distrito de Guápiles nace Paula Zamora Rodríguez, hija de un Ingeniero Agrónomo y una Administradora de Empresas, quienes siempre se enfocaron en brindar a sus hijos una educación de calidad a pesar de las dificultades económicas que se presentaron durante la época colegial y universitaria.

Durante la adolescencia, Paula soñaba con una carrera que le mostrara un poco de todas la disciplinas con el fin de aprender y poder desempeñarse en diferentes ámbitos, por esta razón cuando tuvo la oportunidad de ver los planes de estudios de las diferentes universidades no dudo en participar para ingresar en la carrera de Ingeniería en Seguridad Laboral e Higiene Ambiental del Instituto Tecnológico de Costa Rica, el hecho de brindarse esta carrera en una universidad con reconocimiento nacional fue motivación para investigar un poco más sobre la profesión y enrutarse hacia el camino de la salud y seguridad laboral.

Durante el proceso de formación, descubrió que el área de Higiene Ocupacional abre las puertas para nuevos conocimientos en diversos ámbitos, razón por la cual al enfrentarse a la decisión de cuál sería su temática principal para elaborar un proyecto de graduación, se enfocó específicamente en los agentes ambientales químicos. Tomando en cuenta que su padre formaba parte de los pequeños empresarios que se dedicaban a la agricultura, específicamente producción de palmito de pejibaye, y quién estaban pasando por una dura época en el uso de plaguicidas para el control en la plantación, Paula decide realizar un estudio sobre la exposición dermal para valorar a los pequeños agricultores durante el uso de herbicidas. Estudio que le permitió realizar muestreos en otro tipo de plantaciones, esto después de recibir con orgullo el título académico de Ingeniera en Seguridad Laboral e Higiene Ambiental en Febrero del año 2009. 


\section{Palabras clave}

Exposición dermal; plaguicidas; flores; chayote; palmito

\section{Resumen}

Costa Rica exporta una gran variedad de productos agrícolas, entre los que se encuentran el chayote, el palmito y las flores. El control de plagas se hace a través de diversos mecanismos que incluyen el uso de plaguicidas, a pesar de los efectos que pueden producir en los trabajadores. En este estudio de tipo exploratorio se compararon, en una muestra de productores de flores, chayote y palmito, tres técnicas de exposición dermal: el algoritmo de aproximación de exposición a plaguicidas (AAEP), la de trazador fluorescente (TTF) y la de parches absorbentes (TPA). El AAEP estimó condiciones de exposición hasta ocho veces por encima del nivel de referencia (calculado a partir de las condiciones de trabajo seguras para cada cultivo). La TTF mostró porcentajes medios de exposición en trabajadores de flores y helechos de 13\% (2,4 a 36,6\%); en chayote de $24,8 \%(5,9-51,6 \%$ ) y en palmito de $21 \%$ (19-31\%). Los segmentos corporales con mayor deposición fluorescente fueron las manos (50-68\%), aunque los antebrazos y cuello mostraron porcentajes importantes. Los aplicadores con guantes presentaron deposiciones fluorescentes significativamente más bajas que aquellos que no los usaban. Se observó una asociación significativa entre la deposición fluorescente y el nivel de intensidad por trabajador en los sectores de flores y palmito, con una correlación Spearman $(R=0,86$ y $-0,73)$ respectivamente. EI AAEP no es aplicable a todos los tipos de cultivos ya que las variaciones podrían dar lugar a una subestimación. Es necesario adaptar las herramientas disponibles, con el fin de evaluar la exposición en el sector agrícola.

\section{Key words}

Dermal exposure; pesticides; chayote; flowers; palm hearts

\section{Abstract}

Costa Rica exports a wide variety of agricultural products such as chayote, palm hearts, flowers and ferns. Different methods are used for pest control, including chemical based pesticides, even though they could be damaging the farmer's health. This exploratory study, aimed to compare, in a sample of farmers from each of these crops, three different techniques for dermal exposure assessment: the algorithm quantitative approach method (AQAM), fluorescent tracer and dermal patches. From the algorithmic the intensity levels of exposure were obtained, with fluorescent tracer technique (FTT), deposition of pesticides on workers skin was estimated during application and with absorbent patches the amount of selected pesticides was quantified according to $\mathrm{WHO}$ protocol. The AQAM estimated exposure conditions up to eight times higher compared to the reference level (calculated from safe working conditions for each crop). The FTT showed average percentages of body exposure in flowers and ferns of I $3 \%(2,4-36,3 \%)$ for chayote 24,8\% (5,9-5,.6\%) and palm hearts 21\% (19-31\%). Highest fluorescent tracer deposition body segment were hands (50-68\%), although forearms and neck, showed important percentages. Applicators wearing gloves presented fluorescent depositions significantly lower than those not using protection. Significant association of the fluorescent deposition and intensity level per employee was found for flowers and palm hearts using a Spearman correlation $(R=$ 0.86 and -0.73). The AQAM is not applicable to all types of crops since variations within could lead to exposure sub-estimation. It's necessary to generate our own low-cost tools to assess exposure in the agricultural sector. 


\section{Introducción}

En Costa Rica, para el combate de plagas en los cultivos de palmito, flores, helechos y chayote se utilizan diferentes métodos, incluyendo el control químico a base de piretroides, los cuales podrían causar daños a la salud de los trabajadores agrícolas, ya que algunos de estos productos son neurotóxicos. Un estudio danés puso de manifiesto que en operaciones agrícolas y de horticultura existía un elevado riesgo de la enfermedad de Parkinson, presumiblemente debido a la exposición a los pesticidas neurotóxicos (Tuchsen et al., 2000).

Otros estudios toxicológicos sugieren que estas sustancias también tienen efectos supresores en el sistema inmune y pueden causar daños en los nódulos linfáticos y el bazo. Ente los signos y síntomas por exposición a este tipo de plaguicidas se incluyen sensación de ardor en la cara, dolor de cabeza, náuseas, pérdida del apetito y fatiga en casos leves; además de convulsiones en casos severos (He et al., 1988, Wang et al., 2007, Zhang et al., 199I). El riesgo más común de exposición a plaguicidas lo representa la vía dermal (FAO, 200I), debido a las propiedades físicas y químicas que usualmente posee este tipo de sustancias.

Existen diversos métodos para realizar evaluaciones de la exposición dermal a plaguicidas. El método algorítmico de aproximación cuantitativa (Dosemeci, 2002) fue creado para estimar la exposición de una persona a los plaguicidas a largo plazo y se desarrolló a partir de un estudio efectuado en 58000 aplicadores de plaguicidas en Carolina del Norte y lowa, Estados Unidos.

Por otro lado, la técnica del trazador fluorescente (TTF) permite mostrar de forma visual la zona de contacto entre las sustancias de interés y la piel del trabajador, de manera que la distribución del contaminante se valora según la magnitud e intensidad de las trazas de fluorescencia (Fensky, 1993, Aragón, 2004, Ivancic et al., 2004).

Para cuantificar la cantidad de contaminante que logra alcanzar la piel de la persona se puede emplear el protocolo de la Organización Mundial de la Salud (OMS) sobre parches absorbentes (TPA), que en Costa Rica se ha aplicado para estudios efectuados en plantaciones de banano con el fin de caracterizar la exposición dérmica ocupacional a los plaguicidas (Vaquerano, 1995).
A pesar de que los cultivos de palmito, flores, helechos y chayote tienen varios años de haberse establecido en el país, no se han desarrollado estudios sobre la exposición ocupacional a los plaguicidas en estos sectores.

El presente estudio pretende comparar las tres técnicas de evaluación de exposición dermal, de forma exploratoria, en un grupo piloto de diez pequeños productores de cada uno de estos cultivos. Se espera que la información sea de utilidad tanto a empresarios como a trabajadores para desarrollar de manera segura su labor diaria y que pueda usarse como base para el diseño de estudios posteriores.

\section{Materiales y métodos}

Como grupos piloto se seleccionó una muestra de I 0 fincas de chayote ubicadas en Ujarrás de Cartago, I I productores de palmito de la región atlántica y 10 productores de flores y helechos de las provincias de Alajuela y Cartago. Los productores fueron contactados por medio de llamadas telefónicas y visitas a los cultivos.

Se eligieron los plaguicidas a muestrear según lo que los entrevistados reportaron como de uso común (bifentrina/cihalotrina para chayote, 2,4-D para palmito y bifentrina/deltametrina para flores $y$ helechos).

Se le hizo una entrevista a cada productor $y$, por medio de una encuesta higiénica, se recolectó información sobre las jornadas laborales, el número de empleados, la manipulación de las sustancias utilizadas, las herramientas para la aplicación y el mezclado, las formas de mezclado, el equipo de protección personal y las medidas de higiene personal. Además, se recolectaron datos sobre vigilancia médica y medidas de control en el manejo de plaguicidas, incluyendo los requerimientos del algoritmo de aproximación de exposición a plaguicidas (AAEP). Posteriormente se coordinaron los muestreos, empleando las técnicas de trazador fluorescente y parches absorbentes.

\section{Recolección de datos durante los muestreos}

Mediante la observación no participativa, durante las visitas en el campo se registraron en una bitácora de muestreo los procedimientos utilizados por los trabajadores para realizar las aplicaciones de plaguicidas en las áreas de cultivo. 
Estimación del nivel de intensidad (NI)

de la exposición a plaguicidas

El método algorítmico de aproximación cuantitativa (Dosemeci, 2002) permite evaluar la exposición a plaguicidas a partir de la recolección de información por medio de cuestionarios y la asignación de puntajes a diferentes factores considerados determinantes de exposición. Se presentan dos algoritmos para la estimación de la exposición a plaguicidas: uno general, que considera aspectos relacionados directamente con la exposición durante tareas de aplicación; y uno detallado, que complementa el anterior con información sobre hábitos de higiene, limpieza y control de derrames.

En una primera aproximación para evaluar si alguno de estos algoritmos efectivamente podía ser aplicado a trabajadores en Costa Rica (que trabajan en condiciones y cultivos diferentes a la población de referencia), se propuso la comparación de esta metodología con la de trazador fluorescente, la cual ha sido probada extensamente en esta región. Sin embargo, debido a las limitaciones de la TTF para contemplar el impacto de los hábitos de higiene, limpieza y derrame tal como se plantean en el algoritmo completo, se utilizó el general para poder hacer una correlación más cercana con la población en estudio.

Se estimó el $\mathrm{NI}$ de la exposición a plaguicidas para cada finca a partir de la información consignada en la encuesta higiénica y las bitácoras de muestreo, según la siguiente fórmula:

$$
\mathrm{NI}=(\text { Mezcla }+ \text { Aplicación }+ \text { Reparación }) * \text { EPP }
$$

El método algorítmico establece valores para cada una de las categorías incluidas en el cuestionario; los puntajes fueron asignados según los tipos de tareas realizadas en la finca de cada productor.

Con el fin de establecer en qué situación se encontraban los productores en estudio con respecto a la exposición a plaguicidas, se comparó el $\mathrm{NI}$ obtenido en cada finca contra un valor de referencia, el cual se calcula según ciertos valores establecidos por el método que indican la mayor protección en el manejo de plaguicidas. El valor de referencia se calculó considerando que todas las personas entrevistadas utilizaban bomba de espalda para la aplicación de plaguicidas.

\section{Trazador fluorescente}

Para las evaluaciones con el trazador fluorescente se empleó la modificación propuesta por Aurora Aragón (2004) de la técnica de video imagen para evaluar la exposición (VITAE, por sus siglas en inglés) de Fensky (1993). La técnica establece un sistema de valoración visual según la intensidad y extensión de las deposiciones fluorescentes observadas en el sujeto de estudio.

Para la evaluación de las manchas observadas en la piel del trabajador se utilizó una lámpara portátil de luz ultravioleta y Tinopal CBS-X como trazador (a una concentración de $625 \mathrm{mg} / \mathrm{L}$ ). Como cuarto oscuro se acondicionó la bodega de almacenamiento más cercana al sitio de aplicación en cada finca o invernadero.

La metodología de Aragón establece una división del cuerpo en 31 segmentos para evaluar la exposición; sin embargo, para el presente estudio solo se consideraron 27 regiones, ya que no se observaron las regiones anterior y posterior de ambos muslos, glúteos y genitales.

A partir de los valores obtenidos de extensión e intensidad de las manchas observadas con la TTF, junto con el porcentaje correspondiente a cada segmento de la superficie del cuerpo, se calculó un valor del segmento del cuerpo (VSC), el cual se expresa de la siguiente manera:

$$
\operatorname{VSC}=\frac{\% S C * 1 * E}{5}
$$

Donde: \%SC: Porcentaje correspondiente al segmento del cuerpo; I: Intensidad; E: Extensión.

La sumatoria de estos valores, denominada puntaje visual total (PVT), se comparó con el PVT máximo de exposición para cada trabajador y se estimaron los porcentajes de exposición corporal.

\section{Parches absorbentes}

Se utilizó el protocolo estandarizado para el estudio de campo sobre exposición a plaguicidas de la OMS (1986) como base para la construcción y colocación de los parches absorbentes. Los parches fueron formados con gasa de algodón, a partir de cortes de $10 \times 10 \mathrm{~cm}$ y doblándolos de manera que quedasen con un tamaño de $5 \times 5 \mathrm{~cm}$ y con cuatro capas de gasa (adaptación de la propuesta de McDermott, 2004). Los parches se colocaron en las 
siete regiones del cuerpo establecidas por el protocolo (frente, pecho, abdomen, espalda, antebrazo izquierdo, muslo y pierna izquierda).

Una vez finalizada la aplicación del plaguicida, se retiraron las muestras de la piel o vestimenta (haciendo uso de pinzas) y se introdujeron en viales, para posteriormente ser transportadas bajo condiciones de refrigeración hasta el Laboratorio de Higiene Analítica (LHA) del Instituto Tecnológico de Costa Rica (ITCR), donde se efectuó el análisis correspondiente.

\section{Análisis de los datos}

Relación entre el NI y la deposición del contaminante en la piel

El promedio de los porcentajes de exposición corporal para cada finca y cada trabajador fue correlacionado con los $\mathrm{Nl}$ obtenidos a partir del cuestionario mediante gráficos de dispersión y coeficientes de correlación de Spearman.

\section{Estimación cuantitativa de la exposición a 2,4-}

\section{$D$, bifentrina, cihalotrina y deltametrina}

Las muestras obtenidas de la TPA para bifentrina, cihalotrina y deltamentrina se extrajeron con acetona y se analizaron con el método de cromatografía de gases, para calcular la masa en $\mu g$ del plaguicida. Las muestras con 2,4-D se extrajeron con metanol.
Considerando el tiempo de exposición de cada trabajador (min), el área de los parches y las áreas establecidas por el protocolo $\left(\mathrm{cm}^{2}\right)$ para cada parte del cuerpo, se extrapolaron los datos para obtener la masa de contaminante por unidad de tiempo ( $\mu \mathrm{g} / \mathrm{min})$ para cada región, según se muestra a continuación:

masa $(\mu \mathrm{g}) *$ área del sector $\left(\mathrm{cm}^{2}\right)$ área del parche $( \mathrm { cm } ^ { 2 } ) \longdiv { \text { tiempo de exposición (min) } }$

\section{Resultados y discusión}

A partir de la información obtenida de la encuesta aplicada a los pequeños productores, se estimaron, para cada uno de ellos, los $\mathrm{NI}$ de la exposición y se obtuvo un promedio de valores por finca, los cuales se muestran en el cuadro I. Las diferencias se debieron principalmente a que en algunas de las fincas evaluadas existía una persona encargada de hacer la mezcla de plaguicidas, mientras que en otras los aplicadores también debían realizar labores de mezclado de sustancias. Además, en cuanto al tipo de equipo de aplicación utilizado, algunos de los trabajadores incluidos en el estudio aplicaban con bomba de motor (nebulizador), otros lo hacían con bomba manual de espalda y otros con mangueras desde una tanqueta. También hubo diferencias en cuanto al equipo de protección personal utilizado. La mayoría de los trabajadores tenía botas altas de hule, camiseta o camisa de manga larga, pantalón de

Cuadro I. Valores de NI correspondientes al manejo de plaguicidas para floricultores y productores de chayote y palmito.

\begin{tabular}{|c|c|c|c|}
\hline$N^{0}$ Empresa & NI (floricultores) & NI (chayote) & NI (palmito) \\
\hline 1 & 1,8 & 1,2 & 9,5 \\
\hline 2 & 1,8 & 1,4 & 9,5 \\
\hline 3 & 2,0 & 3,5 & 13,3 \\
\hline 4 & 5,4 & 5,0 & 13,3 \\
\hline 5 & 5,4 & 5,6 & 13,3 \\
\hline 6 & 9,0 & 7,2 & 13,3 \\
\hline 7 & 9,0 & 8,8 & 13,3 \\
\hline 8 & 9,0 & 9,6 & 13,3 \\
\hline 9 & 9,0 & 9,8 & 13,3 \\
\hline 10 & 10,0 & 10,4 & 13,3 \\
\hline 11 & -- & -- & 13,3 \\
\hline Promedio & 6,2 & 6,2 & 12 \\
\hline Valor de referencia & 1,9 & 1,3 & 1,9 \\
\hline
\end{tabular}


mezclilla y gorra. Solo en algunos casos utilizaban capa o delantal, guantes y mascarilla o respirador.

También se contemplaron preguntas sobre otros aspectos del trabajo con el fin de tener un mejor panorama de la situación del sector. Se encontró que las reparaciones y el mantenimiento del equipo no son realizados por los aplicadores. Pocas veces los trabajadores reportaron lavado del equipo y cuando lo hicieron, únicamente indicaron enjuagar con agua para retirar los residuos de sustancias químicas.

Otros resultados de la encuesta revelaron que en solo unas pocas empresas se brinda algún tipo de capacitación sobre el manejo y cuidado de sustancias químicas, a través de cursos en el Instituto Nacional de Aprendizaje (INA), con las casas comerciales que venden los productos o con los trabajadores de mayor experiencia. Con respecto a la vigilancia médica, nuevamente solo en pocas empresas

Cuadro 2. Puntajes promedio por segmento (VSC) y porcentaje de deposición del trazador fluorescente para los productores de flores, palmito y chayote evaluados.

\begin{tabular}{|c|c|c|c|c|c|c|c|}
\hline \multirow[b]{2}{*}{$\begin{array}{l}\text { Superficie } \\
\text { del cuerpo }\end{array}$} & \multirow[b]{2}{*}{$\begin{array}{l}\text { Segmento del } \\
\text { cuerpo }\end{array}$} & \multicolumn{2}{|c|}{ Flores y Helechos } & \multicolumn{2}{|c|}{ Chayote } & \multicolumn{2}{|c|}{ Palmito } \\
\hline & & $\begin{array}{l}\text { VSC promedio } \\
\text { (DS) }\end{array}$ & $\begin{array}{l}\text { \% VSC con } \\
\text { respecto al } \\
\text { máximo }\end{array}$ & $\begin{array}{l}\text { VSC promedio } \\
\text { (DS) }\end{array}$ & $\begin{array}{l}\text { \% VSC con } \\
\text { respecto al } \\
\text { máximo }\end{array}$ & $\begin{array}{l}\text { VSC promedio } \\
\text { (DS) }\end{array}$ & $\begin{array}{l}\text { \% VSC con } \\
\text { respecto al } \\
\text { máximo }\end{array}$ \\
\hline \multirow{3}{*}{ Rostro } & Lado derecho & I,5 $(1,0)$ & 25,1 & $3,17(1,12)$ & 54,3 & $0,47(0,5)$ & 8,0 \\
\hline & Frente & $0,87(0,8)$ & 14,9 & $3,31(1,26)$ & 56,5 & $0,70(1,1)$ & 12,0 \\
\hline & Lado izquierdo & $1,6(1,5)$ & 27,5 & $3,13(1,11)$ & 53,6 & $1,12(0,9)$ & 19,1 \\
\hline \multirow{2}{*}{ Cuello } & Anterior & $1,2(1,4)$ & 22,9 & $2,43(1,23)$ & 48,7 & $0,64(0,8)$ & 12,9 \\
\hline & Posterior & $1,1(1,2)$ & 21,3 & $2,84(1,70)$ & 56,9 & $1,20(1,1)$ & 24,0 \\
\hline \multirow{2}{*}{ Tronco } & Anterior & $3,3(5,9)$ & 5,1 & $10,40(8,59)$ & 16,0 & $4,9 \mid(4,2)$ & 7,6 \\
\hline & Posterior & $3,5(6,0)$ & 5,3 & $20,80(18,30)$ & 32,0 & $19,9(19,4)$ & 30,7 \\
\hline \multirow{2}{*}{ Brazo der. } & Anterior & $1,6(2,8)$ & 16,0 & $2,02(2,12)$ & 20,2 & I,9| $(2,4)$ & 19,1 \\
\hline & Posterior & $1,2(2,0)$ & 11,7 & $1,29(1,85)$ & 12,9 & I,87 $(2,6)$ & 18,7 \\
\hline \multirow{2}{*}{ Brazo izq. } & Anterior & $3,0(2,3)$ & 40,3 & $3,29(3,04)$ & 43,8 & $2,70(2,0)$ & 36,0 \\
\hline & Posterior & $3,7(2,5)$ & 51,5 & $3,25(2,8 I)$ & 43,3 & $2,63(1,9)$ & 35,1 \\
\hline \multirow{2}{*}{$\begin{array}{l}\text { Antebrazo } \\
\text { der. }\end{array}$} & Anterior & $1,6(3,1)$ & 16,0 & $2,17(2,25)$ & 21,7 & $1,69(1,7)$ & 16,9 \\
\hline & Posterior & $1,1(1,8)$ & 10,9 & $1,32(1,94)$ & 13,2 & $0,84(0,8)$ & 8,4 \\
\hline \multirow{2}{*}{$\begin{array}{l}\text { Antebrazo } \\
\text { izq. }\end{array}$} & Anterior & $2,8(3,0)$ & 37,6 & $3,38(2,92)$ & 45,0 & $2,47(2,1)$ & 32,9 \\
\hline & Posterior & $3,4(2,1)$ & 44,8 & $3,12(2,65)$ & 41,6 & $3,13(2,6)$ & 41,8 \\
\hline \multirow{2}{*}{ Mano der. } & Palma & $3,8(2,0)$ & 60,3 & $4,28(2,12)$ & 68,5 & $3,64(1,7)$ & 58,2 \\
\hline & Dorso & $3,6(2,1)$ & 57,1 & $3,85(2,24)$ & 61,6 & $3,11(1,0)$ & 49,8 \\
\hline \multirow{2}{*}{ Mano izq. } & Palma & $4,0(2,3)$ & 63,5 & $4,23(2,18)$ & 67,7 & $3,83(1,9)$ & 61,3 \\
\hline & Dorso & $3,3(2,2)$ & 53,1 & $3,88(2,35)$ & 62,1 & $3,67(0,8)$ & 58,7 \\
\hline \multirow{2}{*}{ Pierna der. } & Posterior & 0,00 & 0,0 & $1,52(4,16)$ & 8,7 & $1,79(2,8)$ & 10,2 \\
\hline & Anterior & 0,00 & 0,0 & $1,52(3,35)$ & 8,7 & $3,97(3,9)$ & 22,7 \\
\hline \multirow{2}{*}{ Pierna izq. } & Posterior & 0,00 & 0,0 & $1,89(4,36)$ & 10,8 & $1,24(2,8)$ & 7,1 \\
\hline & Anterior & 0,00 & 0,0 & $1,40(4,21)$ & 8,0 & $0,93(1,4)$ & 5,3 \\
\hline \multirow{2}{*}{ Pie der. } & Posterior & 0,00 & 0,0 & $0,24(0,83)$ & 2,8 & $0,31(0,6)$ & 3,6 \\
\hline & Anterior & 0,00 & 0,0 & $0,08(0,36)$ & 0,9 & $2,57(3,2)$ & 29,3 \\
\hline \multirow{2}{*}{ Pie izq. } & Posterior & 0,00 & 0,0 & $0,00(0,0$ & 0,0 & $1,24(2,1)$ & 14,2 \\
\hline & Anterior & 0,00 & 0,0 & $0,0(0,00)$ & 0,0 & $3,27(3,3)$ & 37,3 \\
\hline Total & & $46(31)$ & $\begin{array}{c}12,9 \% \\
(2,4-36,6) \%\end{array}$ & 89 (50) & $\begin{array}{c}24,8 \% \\
(5,9-51,6) \%\end{array}$ & 75,8 & $\begin{array}{c}21 \% \\
(18,8-30,8) \%\end{array}$ \\
\hline
\end{tabular}


encuestadas se practican exámenes periódicos específicos para evaluar la exposición a plaguicidas.

Como se puede apreciar en el cuadro I, la comparación efectuada entre los $\mathrm{NI}$ para cada trabajador y el valor de referencia calculado estimó valores hasta ocho veces por encima del nivel deseado de protección en el manejo de plaguicidas.

La TTF se aplicó a un total de 23 trabajadores dedicados al cultivo de chayote, 9 de palmito y 15 floricultores. En el Cuadro 2 se muestran los valores promedio, así como los porcentajes con respecto al máximo, de las deposiciones de trazador fluorescente para cada segmento del cuerpo, obtenidas para floricultores y productores de chayote y palmito, respectivamente.

El máximo valor de PVT (puntaje visual total) que puede obtenerse por la suma de los VSC (valor del segmento del cuerpo) para cada valoración, excluyendo el área posterior de la cabeza, genitales y glúteos, es 453 (Aragón, 2006). Este dato indica que la persona presenta en todo el cuerpo deposiciones de fluorescencia con un valor de cinco tanto en extensión como en intensidad. No obstante, en el estudio no se evaluó el área de los muslos, por lo que el puntaje se redujo a 357,6 como máximo de exposición.

Esta técnica mostró un porcentaje promedio de exposición corporal para productores de chayote de $24,8 \%$ (5,9-51,6\%), para productores de palmito de $21 \%$ (| 8,8-30,8\%), mientras que para los floricultores se tuvo un promedio de $12,9 \%$ (2,4-36,6\%). Es posible observar que en todos los casos las áreas más expuestas son las manos, lo que concuerda con investigaciones previas (Curwin et al., 2003, Machera et al., 2003).

Para aquellos floricultores que usaron guantes se reportó un promedio del $36 \%$ de las manos con trazador, contra un $84 \%$ de deposición en aquellos que no los usaron. En el caso de los productores de chayote, estos porcentajes fueron del $29 \%$ para quienes se protegían sus manos, contra un $88 \%$ para

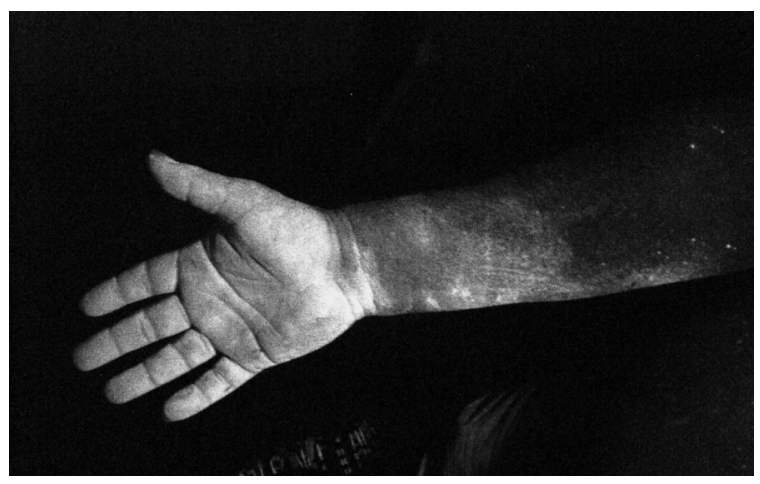

la)

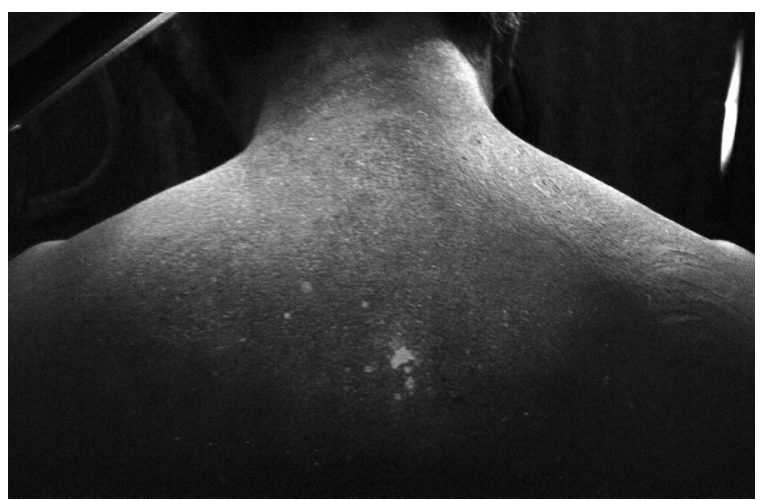

Ic)

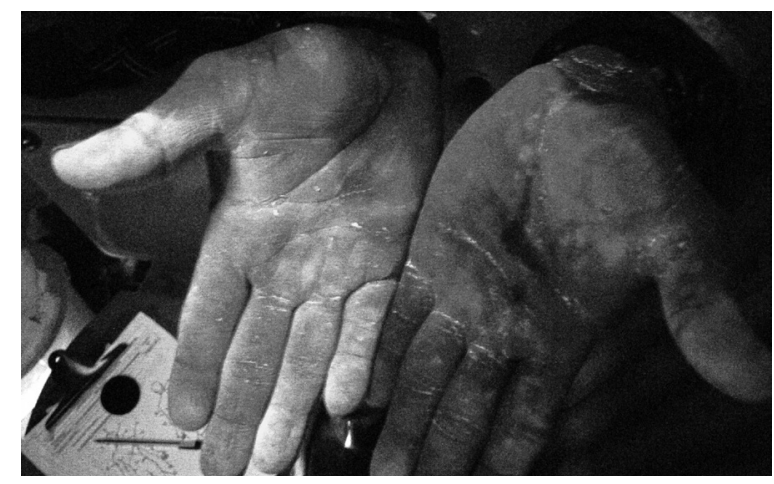

Ib)

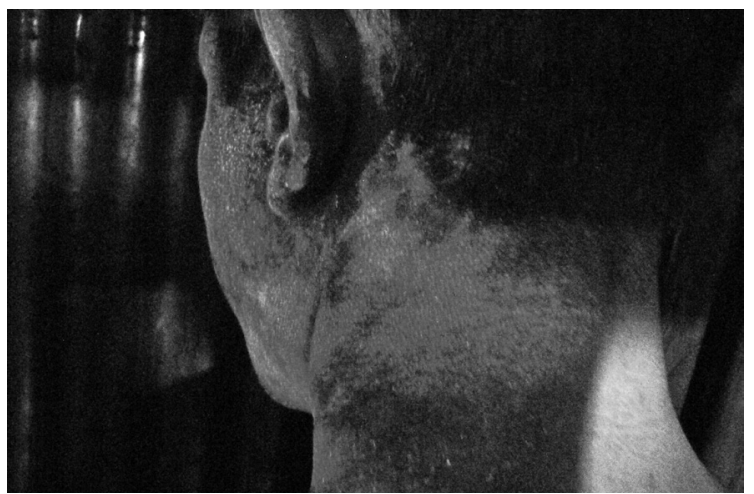

Id)

Figura I: Deposiciones de trazador fluorescente sobre: Ia y lb) manos, Ic) espalda, Id) cuello y rostro. 


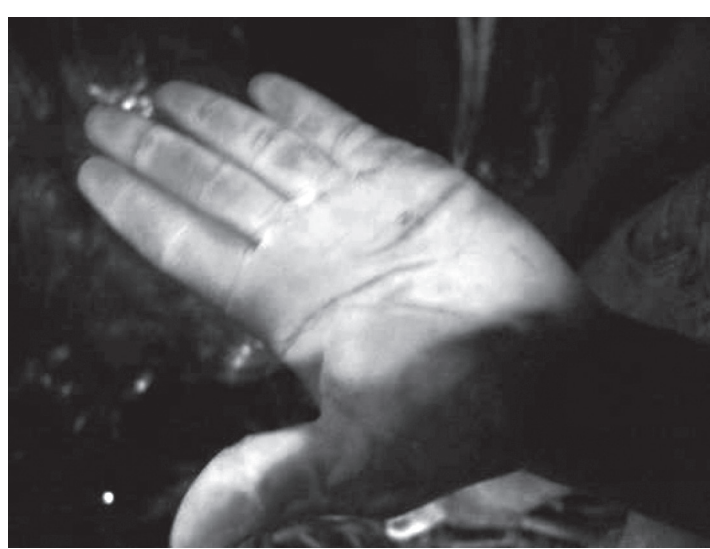

2a)

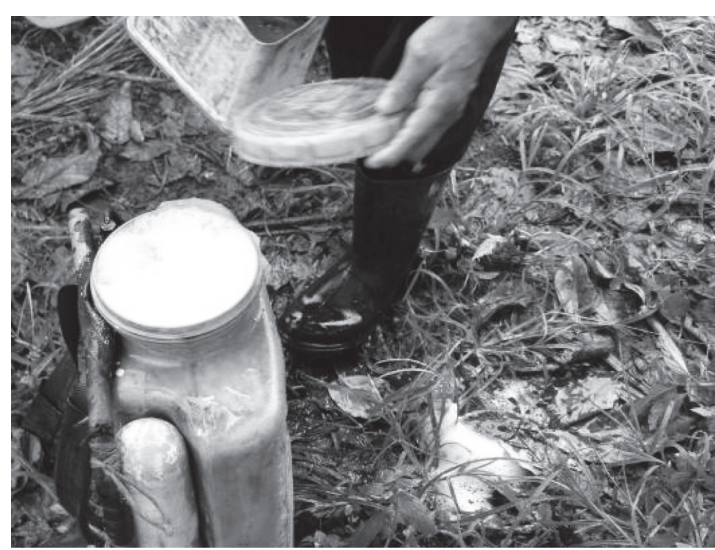

2c)

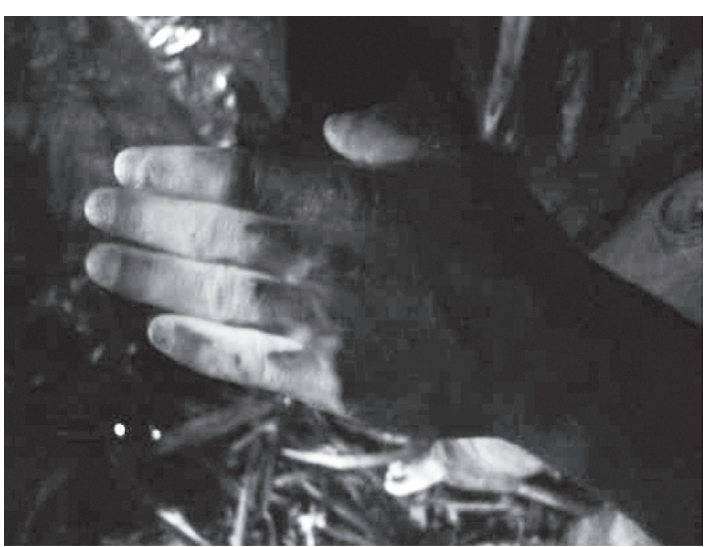

2b)

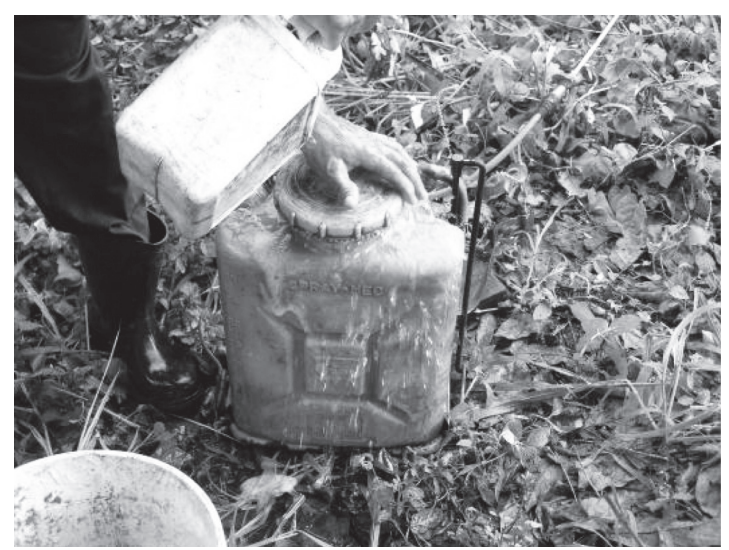

2d)

Figura 2. Deposición fluorescente en manos y prácticas agrícolas inadecuadas de los productores de palmito.

los que no lo hacían. En ambos casos se realizó una prueba de comparación de medias y se demostró, con un 95\% de confianza, que las deposiciones fluorescentes fueron significativamente diferentes entre ambos grupos.

Es importante destacar que, según la información recolectada en las bitácoras de muestreo, los mayores porcentajes de deposición fluorescente se presentaron en aquellos trabajadores que debían preparar o trasvasar la mezcla, así como en aquellos que aplicaban con boquilla tipo campana. Se observó que una práctica frecuente entre los trabajadores evaluados era introducir las manos, sin guantes, en la mezcla (figura 2). Además, algunos de ellos comían en el sitio sin lavarse las manos. También se advierte que el rostro y el cuello, en el caso de los productores de chayote, presentaron valores altos (ver figura I), debido probablemente a que estas regiones se encontraban descubiertas en la mayoría de los tra- bajadores y algunos se frotaban con sus manos el rostro para secarse el sudor.

Las deposiciones en el antebrazo y el cuello de los floricultores pueden atribuirse a la forma de aplicación, pues en muchos casos se observó a los trabajadores colocar la manguera con plaguicida (proveniente de bombas estacionarias) sobre estas áreas del cuerpo para transportarla a través del cultivo (figura 3a). También se observó una importante cantidad de trazador en el área del rostro, muy probablemente por la forma de aplicación, ya que una parte de los floricultores utilizaba mangueras con más de una boquilla (figura 3b), lo que generaba una gran cantidad de aerosol que pudo alcanzar esta región. La mitad de las empresas carecía de protección adecuada para esta zona.

Además, se observó que, dependiendo de la altura del cultivo, era posible que hubiera contacto entre los trabajadores y las hojas impregnadas con el 


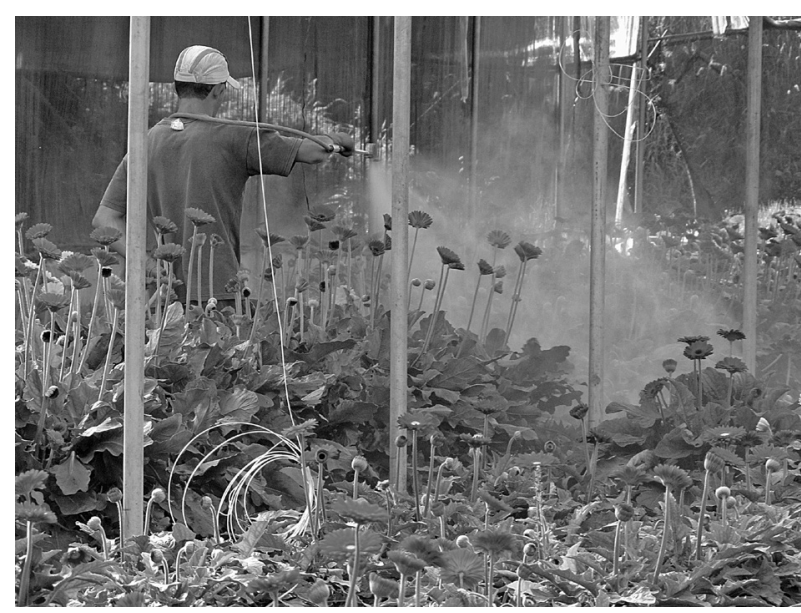

3a)

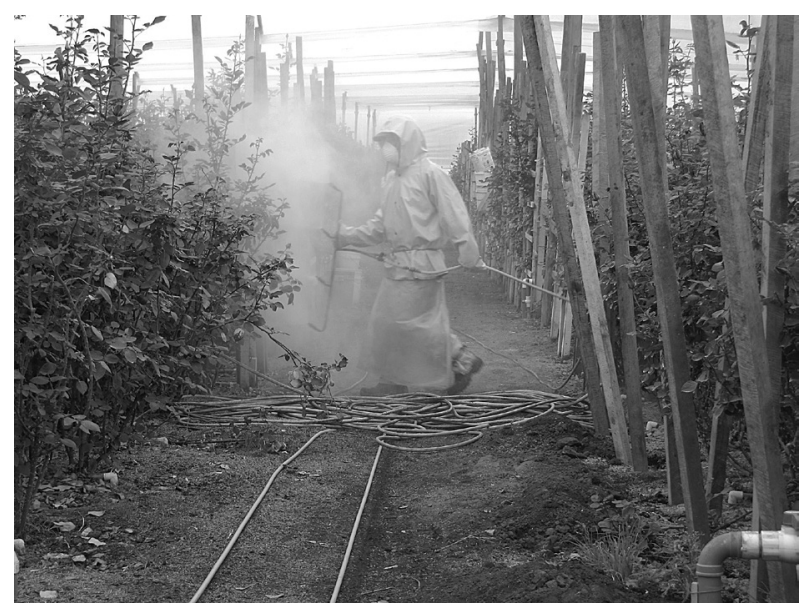

3b)

Figura 3. Modos de aplicación de plaguicidas en cultivos de flores y helechos.

plaguicida durante y al finalizar la aplicación, lo que podría aumentar la exposición. Se reporta en la revisión bibliográfica que entre los factores que pueden influir en la exposición se encuentran la cantidad, la frecuencia y la intensidad del contacto entre la piel y el cultivo (Brouwer et al., 1992).

Caso contrario ocurrió en las extremidades inferiores, para las cuales se reportaron porcentajes muy bajos de deposición del trazador, ya que las botas que portaban los trabajadores les protegía hasta las rodillas, a pesar de que en algunos casos se encontraban en mal estado o rotas.

Los trabajadores que presentaron la menor deposición de trazador fluorescente y a los que también les fue asignado el menor valor de $\mathrm{NI}$, fueron aquellos que sí utilizaban guantes, respirador y gabacha hasta las rodillas. Además, aplicaban desde una tanqueta y caminaban hacia atrás o de lado para evitar que el rocío los impactara de forma directa.

Para determinar si los NI obtenidos a partir de la encuesta podían relacionarse con los datos obtenidos de exposición dermal a través de la TTF, se efectuó una correlación entre ambos para los tres tipos de cultivos. El resultado que se obtuvo fue un coeficiente de correlación de Spearman $(\alpha=0,05)$ de $0,53(p=0,0 \mathrm{I})$ para los productores de chayote, de $-0,73(p=0,02)$ para los productores de palmito y de 0,87 ( $p=0,00 \mathrm{I})$ para los floricultores, como se observa en las figuras 4, 5 y 6 . Esto indica que la dependencia entre ambas variables está ligada al tipo de cultivo y a los mecanismos de aplicación que se utilizan en cada uno.

Para el caso del cultivo de palmito los resultados muestran una correlación negativa, la cual se puede explicar, entre otros factores, porque para este cultivo en particular el herbicida se aplica contemplando que se debe tener cuidado para no dañar los brotes nuevos de las plantas que se encuentran entre la maleza. Es posible asumir que los cuidados que se tienen durante la aplicación son más estrictos que los que siguen para el resto de las prácticas agrícolas evaluadas en el cuestionario, lo cual resulta en que, a pesar de los altos valores de nivel de intensidad calculados, la exposición dermal sea baja.

Para los casos de flores y chayote, la correlación encontrada es débil pero positiva, por lo que en términos generales al aumentar el NI detectado por medio del cuestionario, la deposición del contaminante en la piel es mayor.

A pesar de que la cantidad de trabajadores incluidos en el estudio no es representativa del sector, el resultado obtenido permite suponer que no es posible utilizar el algoritmo general como herramienta para estimar la exposición de los trabajadores en todos los tipos de cultivo.

Cuando se analizan los aspectos contenidos en el cuestionario, se observa que quedan por fuera variables que inciden directamente en la cantidad de plaguicida que puede entrar en contacto con la piel del trabajador durante las aplicaciones en 


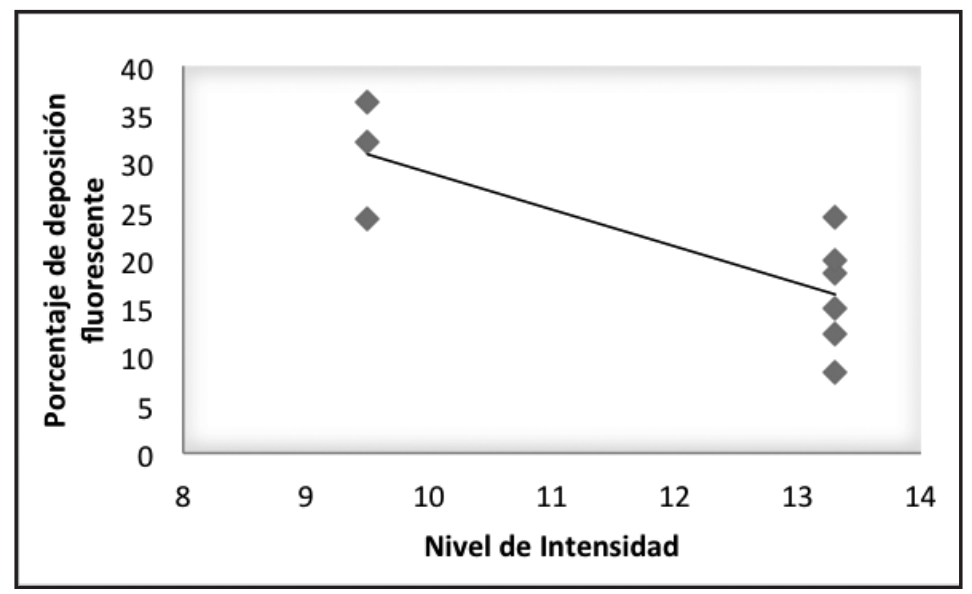

Figura 4. Relación entre los porcentajes de deposición y los NI por trabajador para los productores de palmito.

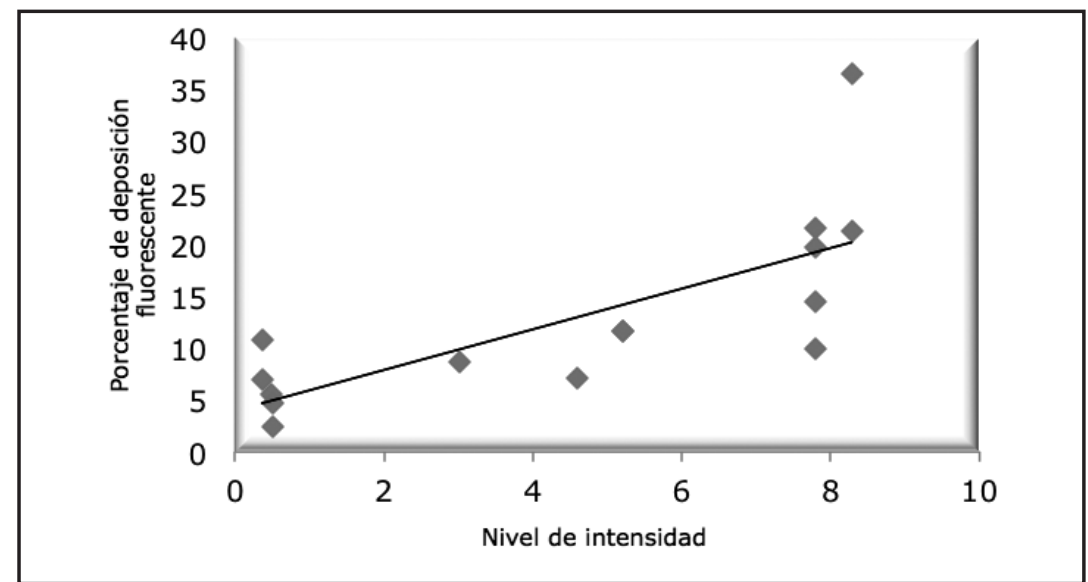

Figura 5. Relación entre los porcentajes de exposición y los NI por trabajador para los floricultores.

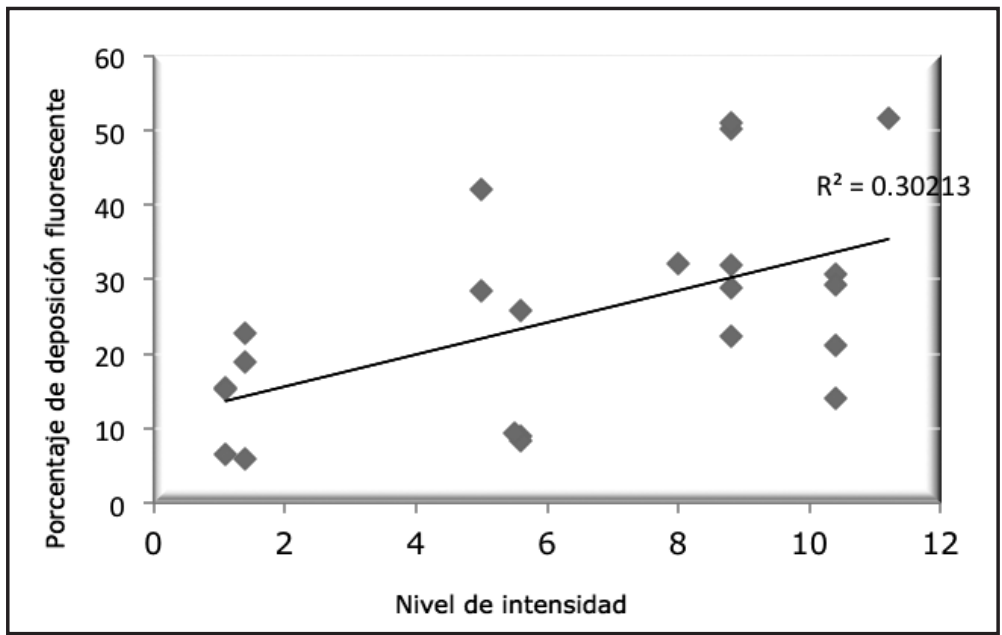

Figura 6. Relación entre los porcentajes de exposición y los NI por trabajador para los productores de chayote. 
campo. Algunos de los aspectos observados incluyen el hecho de que el equipo de aplicación esté contaminado en su parte exterior durante la aplicación (varios trabajadores llenan la bomba hasta que la espuma se rebalse), daños en el EPP (como el deterioro o desgaste de las botas y los guantes), condiciones de higiene durante la manipulación de las sustancias, técnica y dirección de aplicación (para el chayote se aplica hacia arriba) y condiciones ambientales presentes durante las tareas, entre otros.

Además de emplear la TTF, se evaluó mediante la TPA la exposición a 2,4-D (palmito), bifentrina y/o cihalotrina (chayote), bifentrina y/o deltemetrina (flores) en trabajadores según cada tipo de cultivo. El objetivo de realizar un muestreo con parches fue estimar la cantidad de una sustancia particular que se puede depositar en la ropa o la piel durante una determinada actividad. Los parches actúan como muestreadores pasivos que recolectan el contaminante de interés durante el periodo de exposición.

Una de las mayores desventajas del muestreo con parches es que solo estima la cantidad de sustancia depositada en un área particular del cuerpo y se asume que la contaminación está uniformemente distribuida sobre toda la región representada por el parche. Dado que un parche solo representa una proporción relativamente pequeña de cada área en particular, la extrapolación podría llevar a una subestimación en caso de que todas las gotas del contaminante se depositen fuera del parche durante una aplicación en aerosol, o a una sobreestimación en el caso de que el contaminante se concentre sobre el parche (Soutar et al., 2000).

Para contrarrestar estas limitaciones, se combinó el muestreo de parches con el de trazador fluorescente (Kromhout, 1999), de modo que se contara con información sobre la distribución de la exposición en los trabajadores que permitiera una mejor interpretación de los resultados obtenidos.

Las tasas de exposición dermal (en $\mu g / \mathrm{min}$ ) para cada región del cuerpo donde se colocaron los parches en los trabajadores de chayote se muestran en el cuadro 4. Se observa que las regiones de mayor exposición fueron los muslos (ya que los parches colocados se encontraban a la altura de la maleza), el antebrazo y la espalda (en esta última, a la altura de la tapa de la bomba).

Para determinar si los datos obtenidos de exposición dermal a través de la TTF podían ser relacionados con la TPA, se sumaron algebraicamente las tasas de exposición dermal de las regiones evaluadas con parches para cada trabajador, se transformaron a unidades de $\mu \mathrm{mol} / \mathrm{min}$ y se efectuó una correlación con los porcentajes de exposición dermal de la TTF, de lo cual se obtuvo un coeficiente de correlación de Spearman de 0,8I $(p=0,2)$ (ver figura 7). Sin embargo, se contó con una cantidad de datos muy limitada, por lo que sería necesario un número mayor de evaluaciones.

Por otro lado, el análisis de las muestras con bifentrina para floricultores, según la TPA, únicamente mostró concentraciones cuantificables para un trabajador en las áreas de antebrazo y pecho.

Cuadro 3. Tasas de exposición dermal a bifentrina y cihalotrina obtenidas con la TPA ( $\mu \mathrm{g} / \mathrm{min}$ ) para los productores de chayote.

\begin{tabular}{|c|c|c|c|c|}
\hline \multirow{2}{*}{ Región } & \multicolumn{2}{|c|}{ Bifentrina } & \multicolumn{2}{c|}{ Cihalotrina } \\
\cline { 2 - 4 } & Finca 3 & Finca 10 & Finca 8 & Finca 9 \\
\hline Frente & ND & 1,98 & ND & ND \\
\hline Pecho & 0,03 & 0,15 & ND & 0,56 \\
\hline Espalda & 0,38 & 0,47 & ND & 5,94 \\
\hline Antebrazo & 5,17 & 3,67 & ND & ND \\
\hline Abdomen & ND & ND & ND & 22,60 \\
\hline Muslo & 4,75 & 12,31 & ND & ND \\
\hline Pierna & ND & 5,37 & ND & \\
\hline
\end{tabular}

ND: No detectable. 


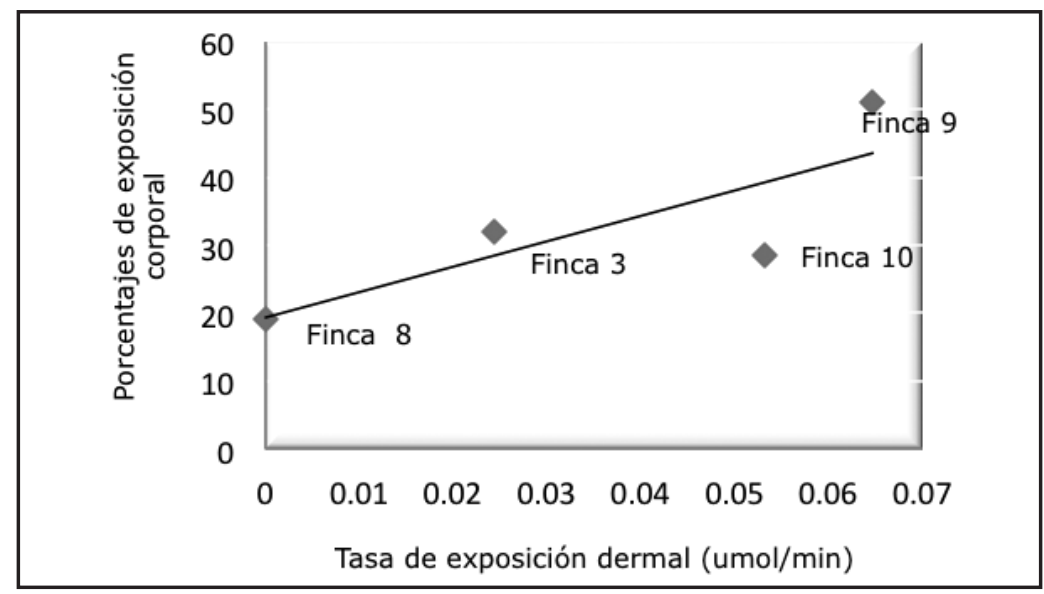

Figura 7. Relación de las técnicas de trazador fluorescente y parches absorbentes para los productores de chayote.

La tasa de exposición dermal a bifentrina para las áreas evaluadas del trabajador fue de 2,52 $\mu \mathrm{g} /$ min. No se obtuvieron resultados detectables para ninguna de las muestras con deltametrina. Estos resultados pueden deberse a que la mayoría de los trabajadores evaluados utilizaba EPP sobre las zonas evaluadas con parches (espalda, abdomen, antebrazo, pierna, pecho y frente), por lo que es razonable que solo se hayan encontrado trazas de los plaguicidas en las diferentes regiones. Además, se resalta el hecho de que los dos datos de bifentrina fueron obtenidos en las áreas de antebrazo y pecho, que a su vez fueron las que, para ese trabajador (el único que no utilizó capa), reportaron los mayores valores de deposición fluorescente, tanto en intensidad como en extensión.

De los cinco trabajadores de palmito evaluados con parches absorbentes, solamente dos presentaron parches con concentraciones. La región con mayor cantidad de herbicida para el trabajador uno fue el antebrazo, lo que se explica por ser una zona cercana a las manos, las cuales presentaron el mayor porcentaje de deposición del trazador fluorescente. El segundo trabajador solo presentó cantidades cuantificables de 2,4-D en la espalda y la frente, muy posiblemente debido a la cercanía del parche con la ubicación de la bomba de espalda y al contacto de las manos con la cara. La deposición de 2,4-D para las áreas expuestas del trabajador uno fue de 56,90 $\mu \mathrm{g} / \mathrm{min}$, mientras que para el trabajador dos fue de $4,19 \mu \mathrm{g} / \mathrm{min}$.

Debido a la poca cantidad de parches que tuvieron valores cuantificables en los floricultores y productores de palmito, no fue posible hacer comparaciones entre la concentración de los agentes químicos en los parches y los valores de deposición fluorescente.

A pesar de que se esperaba una buena correlación entre los resultados de ambas técnicas, debe tenerse en cuenta que existen diferencias atribuibles a condiciones que no pudieron ser controladas durante el estudio. Por ejemplo, la concentración del trazador fluorescente en la mezcla pudo no haber sido la misma que la de los plaguicidas; los plaguicidas presentan propiedades químicas y físicas como solubilidad, volatilidad, viscosidad y polaridad distintas a las del trazador fluorescente; además, las condiciones ambientales, tales como temperatura y porcentaje de humedad relativa altos, pudieron haber afectado la capacidad de la gasa para retener el plaguicida bajo condiciones de excesiva sudoración.

\section{Conclusiones y recomendaciones}

Los valores de los $\mathrm{NI}$ indican que algunos de los trabajadores se encuentran lejos del nivel deseado de protección en el manejo de plaguicidas, según el método algorítmico de aproximación cuantitativa. La comparación efectuada entre los $\mathrm{NI}$ para cada trabajador y el valor de referencia calculado estimó valores hasta ocho veces mayores al nivel deseado de protección en el manejo de plaguicidas.

La TTF confirma que las regiones del cuerpo de los trabajadores que se ven más afectadas son las 
que no están cubiertas (manos, antebrazos, rostro y cuello). Con esta técnica se obtuvo que el promedio de la exposición corporal fue de 24,8\% para los productores de chayote, $21 \%$ para los de palmito y 12,9\% para los floricultores.

Para los aplicadores que usaron guantes, se determinó en sus manos una deposición promedio del 29\% en los productores de chayote y del $36 \%$ para los floricultores; para quienes no los utilizaron se obtuvo $88 \%$ y $84 \%$ respectivamente. En ambos casos se realizó una prueba de comparación de medias y se demostró, con un $95 \%$ de confianza, que las deposiciones fluorescentes fueron significativamente diferentes entre ambos grupos. Esto implica que es importante el uso de guantes como medio para prevenir la exposición dermal.

Los valores para los $\mathrm{NI}$ obtenidos del método algorítmico, los porcentajes de exposición corporal derivados de la TTF y los resultados de la TPA no mostraron una adecuada correlación entre sí en todos los tipos de cultivo evaluados, por lo que se sugiere la aplicación e interpretación de estas tres herramientas de forma complementaria, ya que cada una de ellas aporta información valiosa, considerando variables distintas por las que un trabajador agrícola puede exponerse dermalmente a los plaguicidas.

Se recomienda realizar nuevos estudios que permitan identificar y cuantificar el impacto de otras variables que puedan complementar el método del algoritmo, de modo que se adapte a las condiciones propias de la región y a los tipos de cultivos, dado que la aplicación de un cuestionario para evaluar de manera rápida y semicuantitativa la exposición de trabajadores expuestos a plaguicidas puede ser de gran utilidad para el sector.

\section{Bibliografía}

Aragón, A., Blanco, L., López, L., Lidén, C., Nise, G. \& Wesseling, C. (2004). Reliability of a Visual Scoring System with Fluorescent Tracers to Assess Dermal Pesticide Exposure. Annals of Occupational Hygiene (48) 7, 601-606. Obtenido desde: http://annhyg.oxfordjournals.org/cgi/reprint/48/7/60 I.pdf.

Aragón, A., Blanco, L., Funez, A., Ruepert, C., Lidén, C., Nise, G. \& Wesseling, C. (2006). Assessment of Dermal Pesticide Exposure with Fluorescent Tracer: A Modification of a Visual Scoring System for Developing Countries. Annals of Occupational Hygiene (50) I, 75-83. Obtenido desde: http:// annhyg.oxfordjournals.org/content/50/ //75.full.pdf+html.
Brouwer, R., Marquart, H., De Mik, G. \& Van Hemmen, J. ( 1992). Risk assessment of dermal exposure of greenhouse workers to pesticides after re-entry. Archives of Environmental Contamination and Toxicology (23) 3, 273-280.

Curwin, B., Hein, M., Sanderson, W., Nishioka, M. \& Buhler, W. (2003). Acephate exposure and decontamination on tobacco harvesters' hands. Journal of Exposure Analysis and Environmental Epidemiology (I 3) 203-21 0.

Dosemeci, M., Alavanja, M., Rowland, A., Mage, D., Hoar, S., Rothman, N., Lubin, J., Hoppin, J., Sandler, D. \& Blair, A. (2002). A Quantitative Approach for Estimating Exposure to Pesticides in the Agricultural Health Study. Annals of Occupational Hygiene (46) 2, 245-260. Obtenido desde: http://annhyg.oxfordjournals.org/content/46/2/245.full. pdf+html.

Fensky, R.A. (1993). Dermal exposure assessment techniques. Annals of Occupational Hygiene (37) 6, 687-706.

Food and Agriculture Organization (FAO). (200I). Guidelines on Good Practice for Ground Application of Pesticides. Obtenido desde: http://www.fao.org/docrep/006/Y2767E/ Y2767E00.HTM.

He, F., Sun, J., Han, K., Wu, Y., Yao, P., Wang, S. \& Liu, L. (1988). Effects of pyrethroid insecticides on subjects engaged in packaging pyrethroids. British Journal of Industrial Medicine (45) 548-55।.

Ivancic,W., Nishioka, M., Barnes, R., Hubal, E., Morara, M.\& Bortnick, S. (2004) Development and Evaluation of a Quantitative Video-fluorescence Imaging System and Fluorescent Tracer for Measuring Transfer of Pesticide Residues from Surfaces to Hands with Repeated Contacts. Annals of Occupational Hygiene (48) 6, 519-532. Obtenido desde: http://annhyg. oxfordjournals.org/cgi/reprint/48/6/5 I 9.pdf.

Kromhout, H., Spruit, O. \& Van Puijvelde, M. (1999). Simultaneous use of the fluorescent tracer and surrogate skin pad methods to improve the assessment of dermal exposure to pesticides. Presented at the AlHA Conference and Exhibition, Toronto, Canada.

Machera, K., Goumenou, M., Kapetanakis, E., Kalamarakis, A. \& Glass, C.R. (2003). Determination of potential dermal and inhalation operator exposure to malathion in greenhouses with the whole body dosimetry method. Annals of Occupational Hygiene (47) I, 6I-70. Obtenido desde: http:// annhyg.oxfordjournals.org/content/47/|/6l.full.pdf+html.

McDermott, H. (2004). Air monitoring for toxic exposures. 2 ed. New Jersey: John Wiley \& Sons Inc.

Organización Mundial de la Salud (OMS). (1986). Protocolo estandarizado para estudios de campo sobre exposición a plaguicidas. México.

Soutar, A., Semple, S., Aitken, R. \& Robertson, A. (2000). Use of patches and whole body sampling for the assessment of dermal exposure. Annals of Occupational Hygiene (44) 7 , 5 | |-5 | 8. Obtenido desde: http://annhyg.oxfordjournals.org/ content/44/7/5 | I.full.pdf+html. 
Tüchsen, F. \& Jensen, A. (2000). Agricultural work and the risk of Parkinson's disease in Denmark, 1981-1993. Scandinavian Journal of Work, Environment and Health. (26) 4, 359-362.

Vaquerano, B. (1995). Caracterización de la exposición ocupacional dermal en una plantación bananera en Costa Rica. Tesis Mag. Sc, Universidad de Costa Rica, San José.

Wang, D., Kamijima, M., Imai, R., Suzuki, T., Kameda, Y., Asai, K., Okamura, A., Naito, H., Ueyama, J., Saito, I., Nakajima,T., Goto,
M., Shibata, E., Kondo, T., Takagi, K., Takagi, K. \& Wakusawa, S. (2007). Biological Monitoring of Pyrethroid Exposure of Pest Control Workers in Japan. Journal of Occupational Health (49) 509-5 I4. Obtenido desde: http://joh.med.uoehu.ac.jp/pdf/E49/E49_6_I0.pdf.

Zhang, Z., Sun, J., Chen, S., Wu, Y. \& He, F. (199I). Levels of exposure and biological monitoring of pyrethroids in spraymen. British Journal of Industrial Medicine (48) 82-86. 